\title{
Diet-Induced and Age-Related Changes in the Quadriceps Muscle: MRI and MRS in a Rat Model of Sarcopenia
}

\author{
Claudia Fellner ${ }^{\mathrm{a}}$ Fritz Schick $^{c}$ Robert Kob ${ }^{d}$ Christine Hechtl ${ }^{\mathrm{a}}$ \\ Marianne Vorbuchner ${ }^{\mathrm{e}}$ Roland Büttner ${ }^{\mathrm{b}}$ Okka W. Hamer ${ }^{\mathrm{a}} \quad$ Cornel C. Sieber $^{\mathrm{d}, \mathrm{f}}$

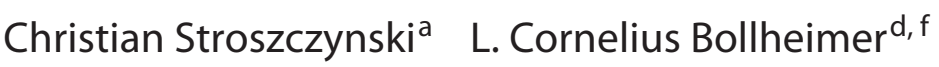 \\ ${ }^{\mathrm{a}}$ Institute of Radiology and ${ }^{\mathrm{b}}$ Department of Internal Medicine I, University Hospital Regensburg, Regensburg, \\ 'Section of Experimental Radiology, Department of Diagnostic and Interventional Radiology, Eberhard-Karls \\ University, Tübingen, ${ }^{d}$ Institute for Biomedicine of Aging, Friedrich-Alexander-Universität, Erlangen-Nürnberg, \\ eSiemens Healthcare Sector, Erlangen, and f Department of General Internal Medicine and Geriatrics, St. John of God \\ Hospital, Regensburg, Germany
}

\section{Key Words}

Magnetic resonance - Sarcopenia · Aging · Muscle ·

Fat quantification

\begin{abstract}
Background: Knowledge about the molecular pathomechanisms of sarcopenia is still sparse, especially with regard to nutritional risk factors and the subtype of sarcopenic obesity. $\mathbf{O b}$ jective: The aim of this study was to characterize diet-induced and age-related changes on the quality and quantity of the quadriceps muscle in a rat model of sarcopenia by different magnetic resonance (MR) techniques. Methods: A total of 36 6-month-old male Sprague-Dawley rats were randomly subdivided into 2 groups and received either a high-fat diet (HFD) or a control diet (CD). At the age of 16 months, $15 \mathrm{HFD}$ and 18 $C D$ rats underwent $M R$ at 1.5 T. T1-weighted images as well as T2 relaxation time maps were acquired perpendicular to the long axis of the quadriceps muscles. Maximum cross-sectional area (CSA) of the quadriceps muscle was measured on T1weighted images, and $\mathrm{T} 2$ relaxation times of muscle were as-
\end{abstract}

sessed in a region without visible intramuscular fat ( $\mathrm{T} 2_{\text {lean muscle }}$ ) and across the complete CSA ( $\mathrm{T} 2$ muscle). Furthermore, ${ }^{1} \mathrm{H}-\mathrm{MR}$ spectroscopy was performed to evaluate the relative lipid content of the quadriceps muscles. These measurements were repeated 5 months later in the surviving $8 \mathrm{HFD}$ and $14 \mathrm{CD}$ rats. Results: HFD rats revealed significantly decreased CSA and CSA per body weight (BW) as well as prolonged $\mathrm{T} 2$ relaxation times of muscle. A higher weight gain (upper tertile during the first 6 months of diet in CD rats) resulted in a significant change of $2_{\text {muscle, }}$ but had no relevant impact on CSA. Advancing age up to 21 months led to significantly decreased BW, CSA and CSA/BW, significantly prolonged $\mathrm{T} 2$ muscle and $\mathrm{T} 2$ lean muscle and enlarged lipid content in the quadriceps muscle. Conclusions: In an experimental setting a chronically fat-enriched diet was shown to have a relevant and age-associated influence on both muscle quantity and quality. By translational means the employed MR techniques give rise to the possibility of an early detection and noninvasive quantification of sarcopenia in humans, which is highly relevant for the field of geriatrics.

(c) 2014 S. Karger AG, Basel

\section{KARGER}

E-Mail karger@karger.com www.karger.com/ger
(C) 2014 S. Karger AG, Basel

0304-324X/14/0606-0530\$39.50/0
Claudia Fellner

Institute of Radiology, University Hospital Regensburg

Franz-Josef-Strauss-Allee 11

DE-93053 Regensburg (Germany)

E-Mail claudia.fellner@ukr.de 


\section{Introduction}

Sarcopenia denotes an excessive decline of skeletal muscle mass and strength with advancing age [1-4]. Being one of the major causes of disability and frailty in the elderly, sarcopenia is a critical issue in an aging society and is, therefore, of major concern in contemporary geriatrics.

Many different underlying causes for sarcopenia have been described in the literature, ranging from disuse atrophy and nutritional imbalances to neurodegenerative diseases [5]. Inflammation and excess of adipocytokines evoked by obesity were identified as risk factors for the loss of muscle mass in the last years [6]. Furthermore, it has been speculated that a surplus of dietary fatty acids could also induce sarcopenia by lipotoxic effects even in the absence of obesity. Thereby, direct induction of apoptosis, autophagy and proteolysis in the myotubes are proposed mechanisms [7]. Furthermore, fatty acids may also interfere with the glucose-dependent $\mathrm{PKB} / \mathrm{Akt}$ signaling and the energy-sensing AMPK pathways, leading to deregulation of mitochondrial biogenesis and protein synthesis [8]. Taken together, diets with a high amount of fatty acids appear to be important for the development and progression of an excessive loss of muscle mass.

Among other parameters of physiologically relevant muscular changes in sarcopenia, muscle loss is primarily monitored by the measurement of the muscle volume or maximum cross-sectional area (CSA) in magnetic resonance (MR) images. In a human study the CSA of the thighs exhibited a good correlation with muscle strength [9]. Furthermore, the CSA of the quadriceps muscle of healthy and mildly and severely sarcopenic men differed significantly from each other [10]. Nonetheless, it has been stated that the decline of muscle strength is underestimated - and therefore not sufficiently covered - by measuring the CSA of a muscle [11]. This fact underlines the necessity for other parameters of muscle quality in studying and diagnosing sarcopenia.

MR imaging (MRI) and spectroscopy (MRS) have been proposed as noninvasive methods to describe agerelated changes of body composition and to quantify body fat distribution [12-14]. In particular, intramyocellular lipid (IMCL) content has been studied as an energy storage within the muscle and in relation to insulin sensitivity [15-19]. Up to now, most MR-based studies focused on age-associated changes, obesity or diet-induced changes in cross-sectional studies [12, 18-23]. Analysis of the $\mathrm{T} 2$ relaxation time and the lipid fraction was performed in order to reveal potential nutritional lipotoxic effects that could play a role in decreasing muscle volume, as recently shown in a pilot study [8].

Against this background, the aim of the present study was to assess diet-induced effects on muscle quantity and quality during aging with MRI and MRS in a rodent model, employing a species with a relatively short life span. For this purpose we have chosen a rat model in a controlled experimental setting as it has several advantages compared with a similar study in humans: the dietary concept can be strictly followed, the influencing factors can be more clearly defined, the comparison with a control group is much easier and observation along a considerable fraction of lifetime is possible.

\section{Materials and Methods}

Animals and Dietary Procedure

A total of 36 male Sprague-Dawley rats were purchased (Charles River Laboratories, Sulzfeld, Germany) at the age of 6 months and included in the study without further selection or conditioning. In the first 6 months of life all animals got standardized species-appropriate husbandry.

The rats were subdivided into 2 groups receiving either a highfat diet (HFD, $n=18,43$ energy percent of neutral fat, based on lard and corn oil) or a control diet (CD, $n=18,25$ energy percent of neutral fat; Altromin, Lage, Germany). The HFD contains exactly double the amount of each fatty acid species as the CD. For partial compensation the polysaccharides were reduced by $37 \%$, leading to an approximately $14 \%$ surplus of metabolizable energy in the HFD. The concentration of all other components including disaccharides and amino acids were identical in both chows. All rats were given ad libitum access to water and food and were housed in groups of 3 rats per cage at a constant room temperature of $20^{\circ} \mathrm{C}$ and on a 12 -hour light-dark cycle until the end of the experiment.

MRI and MRS examinations were performed at the age of 16 and 21 months with an identical protocol for all animals.

All animal procedures were approved by the local animal rights committee and were in compliance with the German law on animal protection.

\section{MR Examination}

The animals were anesthetized by intraperitoneal injection of pentobarbital (Narcoren ${ }^{\circledR}$, Merial, Hallbergmoos, Germany), oxygen was delivered via a mask during the MR examination and the animals were covered with a blanket to avoid cooling.

MRI and ${ }^{1} \mathrm{H}$-MRS were performed on a 1.5-tesla clinical scanner (Magnetom Avanto; Siemens Healthcare, Erlangen, Germany) using an 8-channel array coil designed for human knee examinations. The rats were examined in prone position with the hind limbs extended backwards and T1-weighted spin echo (SE) sequences were applied to image the quadriceps muscles of both hind limbs (see fig. 1). Based on sagittal images (fig. 1b), 'coronal' images along the long axis of the quadriceps muscle (fig. 1d) and 'transverse' images perpendicular to the long axis of the quadriceps muscle (fig. 1c) were acquired (repetition time, TR: $450 \mathrm{~ms}$, echo 


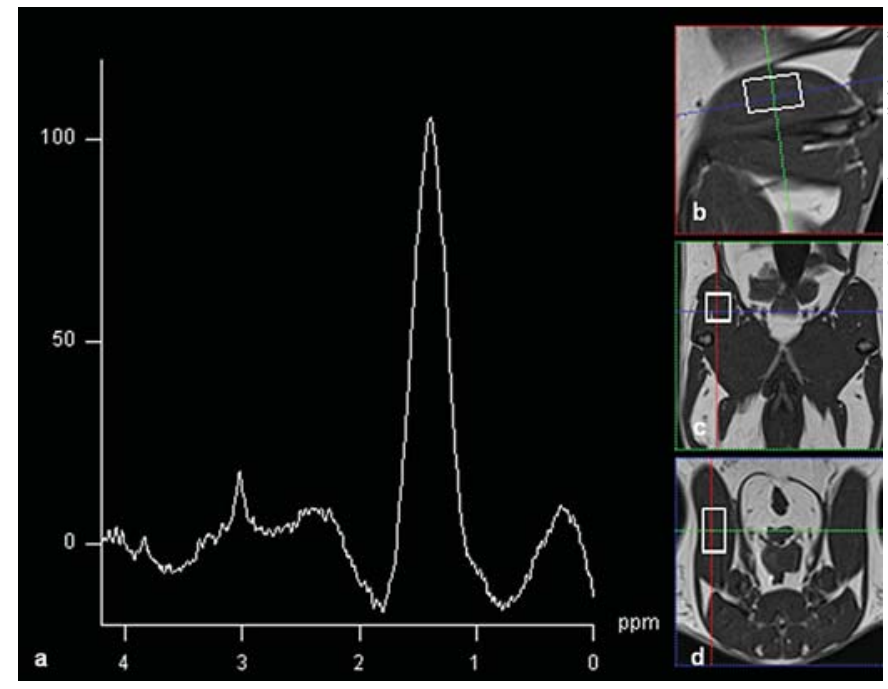

Fig. 1. PRESS spectrum of the right quadriceps muscle in an HFD rat at the age of 21 months (a). Positioning of the voxel in sagittal (b), transverse (c) and coronal (d) orientation within the right quadriceps muscle.

time, TE: $11 \mathrm{~ms}$, voxel size: $0.4 \times 0.4 \times 2.0 \mathrm{~mm}^{3}, 21$ slices, slice gap: $0.2 \mathrm{~mm}$ ). A multiecho SE sequence (TR: 2,000 ms) consisting of 12 echoes (TE: $14-169 \mathrm{~ms}$, voxel size: $0.4 \times 0.4 \times 2.0 \mathrm{~mm}^{3}$ ) was also applied perpendicular to the long axis of the quadriceps muscles and color-coded T2 maps were calculated by an algorithm based on monoexponential fitting (fig. 2).

${ }^{1} \mathrm{H}$ single voxel MRS was performed using a point-resolved spectroscopy sequence (PRESS) technique with a TR of $1,500 \mathrm{~ms}$, a TE of $30 \mathrm{~ms}, 192$ averages, and water suppression. Voxels with a constant size of $12 \times 6 \times 7 \mathrm{~mm}^{3}$ were positioned in the right and left quadriceps muscle. Additional spectra without water suppression were recorded from the same volumes (single scan) for fat signal quantification, using water as an internal reference.

\section{Assessment of Biomarkers by MRI and MRS}

The CSA of the quadriceps muscle was measured for both hind limbs using T1-weighted SE images orientated exactly perpendicular to the long axis of the muscle. As strictly symmetrical positioning of both hind limbs was not possible in all rats, 'truly perpendicular' transverse slices were therefore reconstructed from the original transverse slices for each hind limb separately. Maximum CSA was then taken from the slice with the largest CSA. The reproducibility of this method was determined in a pilot study with 1 rat that was measured 3 times in a row - including repositioning of the animal between single measurements. Each MR data set was evaluated 5 times, resulting in 15 results for CSA.

$\mathrm{T} 2$ relaxation times of 'lean muscle' (T2 $\left.2_{\text {lean muscle }}\right)$ were assessed in circular regions of interest (ROIs) within the quadriceps muscle, avoiding regions of visible intramuscular fat. In addition to these values representing lean musculature only, an average T2 relaxation time ( $\left.\mathrm{T} 2_{\text {muscle }}\right)$ was also evaluated for an area comprising the entire CSA (see fig. 2). Both contours were documented to allow comparable positioning in the follow-up examination at the age of 21 months (fig. 2).
MR spectra were postprocessed using the software package offered by the vendor. Processing included subtraction of the water signal (when applicable), Fourier transformation, Hanning filtering with a width of $700 \mathrm{~ms}$, zero filling from a measured vector length of 1,024 to a length of 2,048, baseline correction (6th order polynomial), curve fitting, and manual phase correction for all spectra. Relative lipid content was calculated as 'lipid fraction' = $\left[\mathrm{MRS}_{\text {lipids }} /\left(\mathrm{MRS}_{\text {lipids }}+\mathrm{MRS}_{\text {water }}\right)\right] \times 100$, with $\mathrm{MRS}_{\text {water }}$ being the area under the water signal in the spectrum recorded without water suppression, and $\mathrm{MRS}_{\text {lipids }}$ as the integral signal taking into account all lipid signal contributions between 0.9 and $1.6 \mathrm{ppm}$ and between 1.9 and $2.6 \mathrm{ppm}$.

\section{Statistical Analysis}

Statistical analysis was performed using SPSS 21 (IBM SPSS Statistics, Armonk, N.Y., USA). Kaplan-Meier survival curves were generated for HFD and CD rats, and the log-rank test was applied to compare the survival in both dietary groups. For all MRI and MRS data (CSA, T2 relaxation times and lipid fraction), results of the right and left leg were averaged. All data are given as mean \pm standard deviation within 1 group or subgroup related to age and/ or diet. Reproducibility of CSA measurements was estimated by calculation of the variation coefficient: (standard deviation/mean value) $\times 100$.

To assess diet-induced changes at the age of 16 months, twosided unpaired t testing was applied if normal distribution of data was confirmed by the Kolmogorov-Smirnov test; Levene's test was used to test the homogeneity of variances. A $2 \times 2$ mixed-design ANOVA was used to analyze the influence of diet and age in those rats that survived until the age of 21 months; age served as the within-subject factor and diet as the between-subject factor. Differences with $\mathrm{p}<0.05$ were regarded as statistically significant for all tests.

\section{Results}

Up to the age of 16 months - the time of the first MR examination -3 of 18 HFD rats died (fig. 3). Another 3 HFD animals died before the second MR examination at the age of 21 months. An additional 4 rats had to be sacrificed due to large neoplasms or worsening general health condition according to good animal care practice (UFAW Handbook on the Care and Management of Laboratory Animals, 2010). From the CD group, 2 animals died and another 2 had to be sacrificed before the age of 21 months (fig. 3). Thus, MR was performed at the age of 16 months in 15 HFD and 18 CD rats; the identical MR protocol could be repeated 5 months later in 8 HFD and $14 \mathrm{CD}$ rats. The log-rank test revealed a significantly longer survival of $\mathrm{CD}$ rats compared with HFD rats ( $\mathrm{p}=$ 0.041 ).

The variation coefficient for the assessment of CSA evaluated in 15 consecutive measurements - was $1.77 \%$, indicating very good reproducibility of this method. 

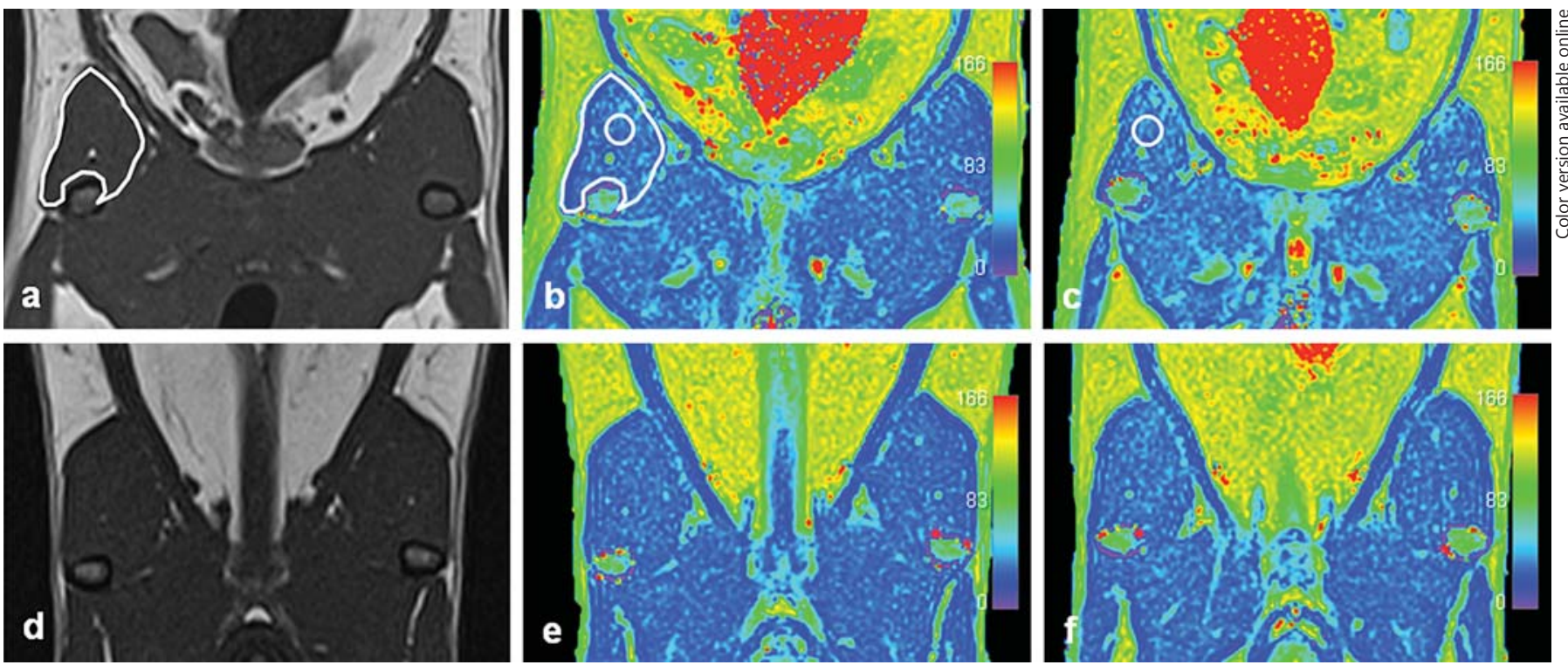

Fig. 2. Longitudinal results of an HFD and a CD rat. T1-weighted image of an HFD (a) and CD rat (d) at the age of 16 months as well as color-coded T2 maps of an HFD rat at 16 (b) and 21 (c) months and $\mathrm{CD}$ rat at $16(\mathbf{e})$ and 21 (f) months. Blue indicates short, green longer, and red very long T2 relaxation time. The white contour in a marks the CSA of the right quadriceps muscle and the ROI for $\mathrm{T} 22_{\text {muscle }}$ in $\mathbf{b}$; the circular ROI for evaluation of $\mathrm{T} 2_{\text {lean muscle }}$ is also shown in $\mathbf{b}$ and $\mathbf{c}$.

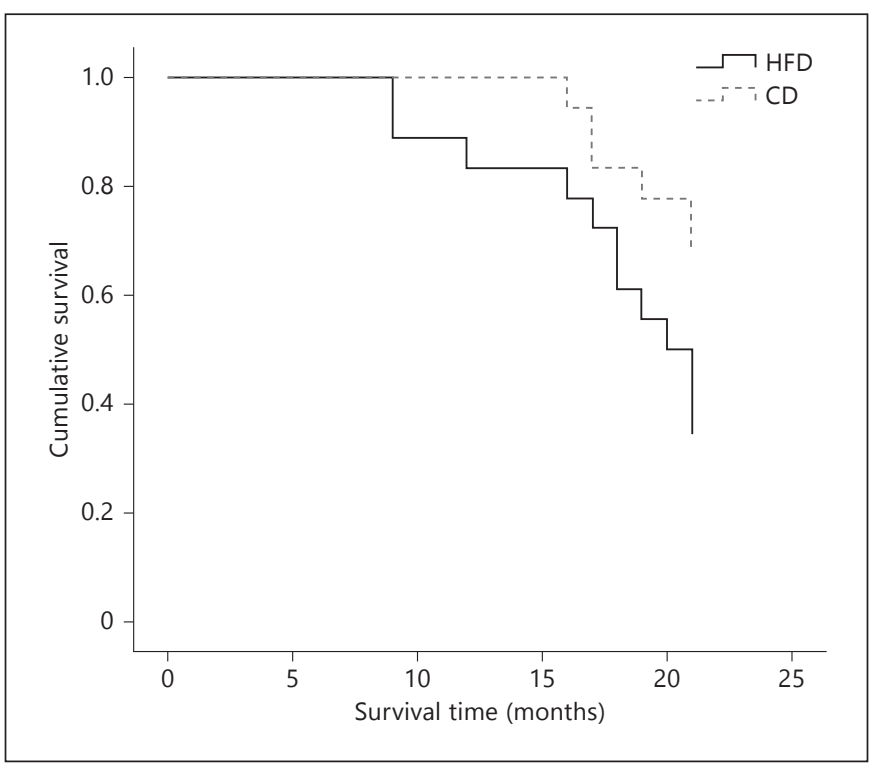

Fig. 3. Kaplan-Meier survival curve for $18 \mathrm{HFD}$ and $18 \mathrm{CD}$ rats.

At the age of 16 months the CSA of the quadriceps muscle was significantly smaller in HFD than in CD rats, while body weight (BW) was similar in both groups (table 1). HFD rats demonstrated prolonged $\mathrm{T} 22_{\text {lean muscle }}$ compared with CD rats.

MR in a Rat Model of Sarcopenia
In order to reveal the effects of BW on muscle parameters the rats were grouped based on an AUC analysis of the weight gain during the first 6 months of diet. With the upper tertile of weight gain in the $\mathrm{CD}$ rats arbitrarily set as threshold, 6 of $18 \mathrm{CD}$ and 9 of 15 HFD rats were classified into the higher weight group (fig. 4). As expected, the BW of upper tertile-weighted HFD and CD rats did not differ significantly from each other, and normal-weight HFD and CD rats showed quite similar BW as well. The CSA of quadriceps muscles was very similar in heavier and normal-weight CD rats, but significantly smaller in normal HFD when compared with normal $\mathrm{CD}$ rats. CSA/BW was significantly reduced in all heavier compared to all normal-weight rats, but also in heavier versus normal CD rats. $\mathrm{T} 2$ muscle - but not $\mathrm{T} 2$ lean muscle was significantly longer in heavier compared with normal-weight rats. Despite missing statistically significant differences between all subgroups (fig. 4), there was a general trend towards longer T2 relaxation time of the muscle in HFD than in CD rats and also in heavier compared with normal-weight rats. The lipid content was very similar in all subgroups - except for a somewhat lower lipid content in normal-weight CD rats.

Longitudinal results were available in $8 \mathrm{HFD}$ and $14 \mathrm{CD}$ rats that survived until the age of 21 months. Figure 2 shows exemplary T2 maps of an HFD and a CD rat - both 


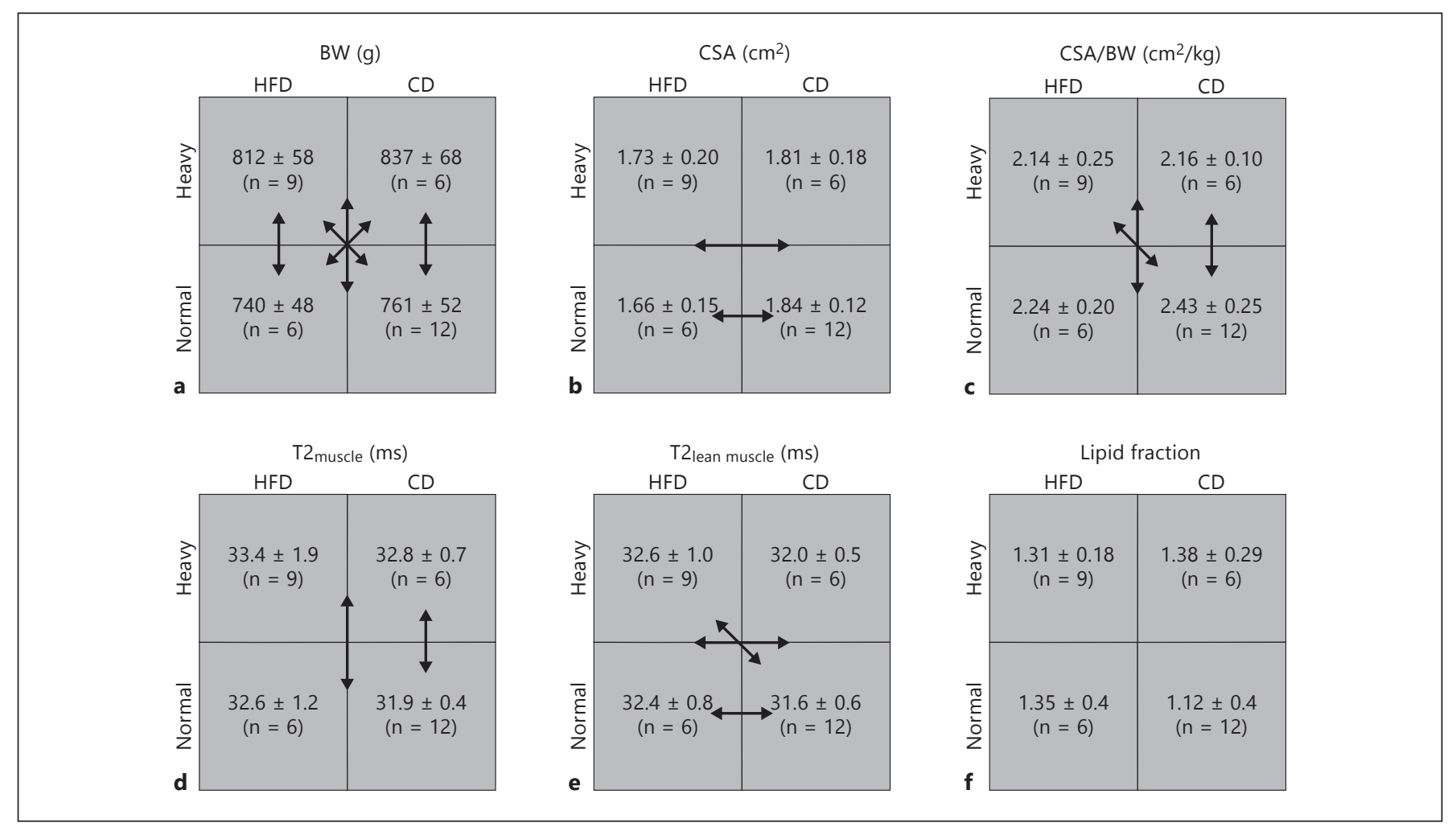

Fig. 4. Higher weight gain (upper tertile during the first 6 months of diet in CD rats) - heavy vs. normal weight - in 16-month-old HFD and CD rats. Influence on BW (a), CSA of the quadriceps muscle (b), CSA/BW (c), T2 relaxation time across the whole CSA of the quadriceps muscle ( $\mathrm{T} 2$ muscle; $\mathbf{d}$ ), T2 relaxation time of the lean quadriceps muscle ( $\left.\mathrm{T} 2_{\text {lean muscle }}\right)$ in a region without visible fat

Table 1. Diet-induced effects on 16-month-old rats

\begin{tabular}{llll}
\hline & $\begin{array}{l}\text { HFD } \\
(\mathrm{n}=15)\end{array}$ & $\begin{array}{l}\mathrm{CD} \\
(\mathrm{n}=18)\end{array}$ & $\mathrm{p}$ \\
\hline $\mathrm{BW}, \mathrm{g}$ & $783 \pm 64$ & $786 \pm 67$ & 0.882 \\
$\mathrm{CSA}, \mathrm{cm}^{2}$ & $1.70 \pm 0.18$ & $1.83 \pm 0.14$ & 0.030 \\
$\mathrm{CSA} / \mathrm{BW}, \mathrm{cm}^{2} / \mathrm{kg}$ & $2.18 \pm 0.23$ & $2.34 \pm 0.24$ & 0.065 \\
$\mathrm{~T} 2$ muscle $\mathrm{ms}$ & $33.1 \pm 1.7$ & $32.2 \pm 0.7$ & 0.066 \\
$\mathrm{~T} 2$ lean muscle, $\mathrm{ms}$ & $32.5 \pm 0.9$ & $31.8 \pm 0.6$ & 0.007 \\
Lipid fraction & $1.33 \pm 0.27$ & $1.20 \pm 0.35$ & 0.288 \\
\hline
\end{tabular}

$\mathrm{p}=\mathrm{p}$ value ( $\mathrm{t}$ test $)$

at the age of 16 and 21 months. Smaller CSA and CSA/BW, prolonged $\mathrm{T} 2_{\text {muscle }}$ and $\mathrm{T} 2_{\text {lean muscle }}$ and increased lipids in HFD compared with CD rats were also found in the collective of the 'survivors' - at the age of 16 as well as at the age of 21 months (fig. 5). A $2 \times 2$ mixed-design ANOVA (e) and lipid fraction (f), based on ${ }^{1} \mathrm{H}$ single voxel spectroscopy. Large (horizontal or vertical) arrows denote statistically significant differences between groups (HFD vs. CD or heavy vs. normalweight rats); small arrows denote statistically significant differences between subgroups.

Table 2. Age- and diet-induced effects on $8 \mathrm{HFD}$ and $14 \mathrm{CD}$ rats at the age of 16 and 21 months

\begin{tabular}{|c|c|c|c|}
\hline & $\mathrm{p}$ (age) & $\mathrm{p}$ (diet) & $\mathrm{p}($ age $\times$ diet $)$ \\
\hline BW & 0.048 & 0.837 & 0.508 \\
\hline CSA & $<0.001$ & 0.006 & 0.448 \\
\hline CSA/BW & $<0.001$ & 0.023 & 0.156 \\
\hline $\mathrm{T} 2_{\text {muscle }}$ & 0.009 & 0.016 & 0.670 \\
\hline $\mathrm{T} 22_{\text {lean muscle }}$ & $<0.001$ & $<0.001$ & 0.903 \\
\hline Lipid fraction & 0.013 & 0.359 & 0.969 \\
\hline
\end{tabular}

$\mathrm{p}=\mathrm{p}$ value $($ ANOVA $)$.

revealed no interaction between age and diet, but several significant effects of age or diet alone (table 2). BW, CSA, CSA/BW and T2 relaxation times, as well as lipid content, were significantly influenced by age. Diet had a significant influence on CSA, CSA/BW and T2 relaxation times. 


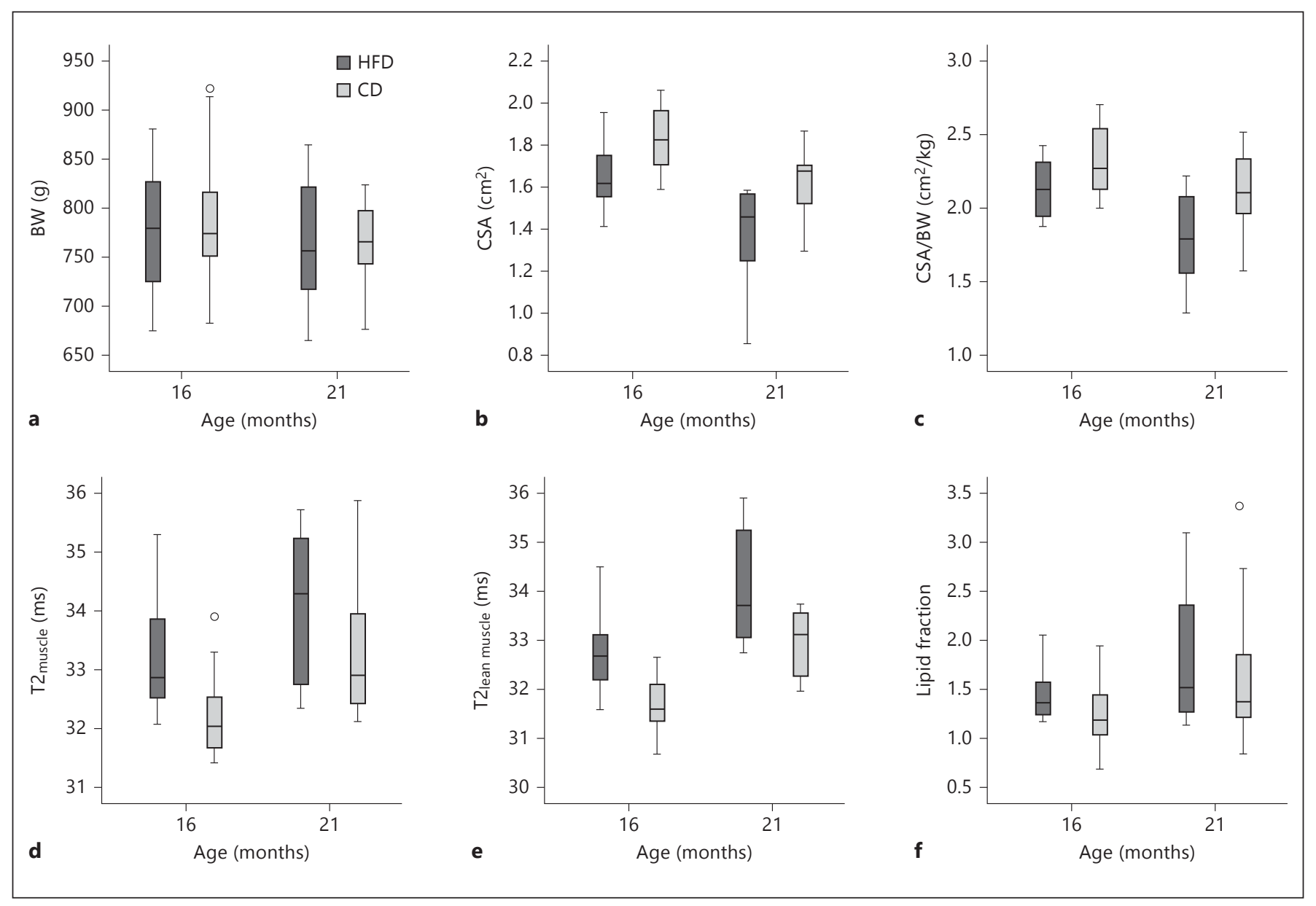

Fig. 5. Boxplots summarizing the longitudinal development in $8 \mathrm{HFD}$ and $14 \mathrm{CD}$ rats at the age of 16 and 21 months. a BW of the animals. $\mathbf{b}$ CSA of the quadriceps muscle. $\mathbf{c}$ CSA/BW. $\mathbf{d}$ T2 relaxation time across the whole CSA of the quadriceps muscle

\section{Discussion}

In the present study, age- and diet-induced changes were investigated in quadriceps muscles because of their prevalence of type 2 muscle fibers which are particularly sensitive to aging effects [24]. While the quadriceps muscle can be easily delineated in the current animal model, further differentiation of single muscles is difficult based on MRI at $1.5 \mathrm{~T}$. The maximum CSA turned out to be a reliable measure of morphological muscle change as long as the image orientation was actually perpendicular to the femur. This requirement could be accomplished in all animals for both hind limbs. Another parameter, CSA/BW, was introduced because of marked interindividual variations of $\mathrm{BW}$ in both dietary groups as well as in the whole collective before allocation. The CSA of the
$(\mathrm{T} 2$ muscle). e $\mathrm{T} 2$ relaxation time of the lean quadriceps muscle $\left(\mathrm{T} 2_{\text {lean muscle }}\right)$ in a region without visible fat. $\mathbf{f}$ Lipid fraction, based on ${ }^{1} \mathrm{H}$ single voxel spectroscopy. Statistical results evaluating the influence of age and diet are presented in table 2. 
in HFD versus $C D$ rats and with increasing age. Besides changes in fat content, changes in water composition have to be taken into account. Hatakenaka et al. [21] reported significantly increased extracellular space (relative to intracellular space) in type 2 muscles of old versus young mice based on histological analysis. While our results of muscle T2 in 16- and 21-month-old rats revealed a significant dependence on age and diet, the lipid content showed an age-related effect but no significant diet-induced effect (table 2). Elevated IMCL has been observed also in obese humans $[18,23]$ and in animal studies with rats undergoing HFD with and without impaired insulin sensitivity [13, 26-28]. Using a whole-body clinical 1.5-tesla scanner for our animal study we were not able to separate IMCL and extramyocellular lipid (EMCL) in the quadriceps muscle of the rat. The ability to separate both peaks depends not only on $\mathrm{B}_{0}$ but also on the voxel size, on the muscle type and on the orientation of its fibers relative to $\mathrm{B}_{0}[16,17]$. IMCL and EMCL have been reported to be well resolvable in human tibialis anterior muscles at 1.5 or $3 \mathrm{~T}[22,23]$ or in ventral and dorsal tibialis anterior muscles of rats at $6.3 \mathrm{~T}$ or above $[13,26-28]$. Since IMCL and EMCL could not be well resolved in our muscle spectra, only total lipid content within the quadriceps muscle was evaluated in our study. However, derived T2 values from different ROIs within cross-sectional images of the hind limbs of the rats provide additional information. Since only very rare amounts of fatty septa within the musculature are visible on the images, one might expect that the fat fraction in $\mathrm{T} 2$ lean muscle is mainly determined by IMCL, while T2 across the whole muscle ( $\left.\mathrm{T} 2_{\text {muscle }}\right)$ also includes fatty infiltrations, i.e. EMCL and intermuscular adipose tissue. As diet-induced effects on $\mathrm{T} 2_{\text {lean muscle }}$ were stronger than effects on $\mathrm{T} 2$ muscle, one might further conclude that these effects mainly result from changes of homogeneously distributed material in the musculature (i.e. water and IMCL) rather than from changes in EMCL and intermuscular adipose tissue.

Age-related changes between the ages of 16 and 21 months revealed a decrease of CSA and CSA/BW as well as an increase of T2 for both diets. Reduced CSA or muscle mass has been reported in older versus younger human cohorts [19, 20, 29]. A relevant increase of muscle T2 with advancing age has been described for human $[12,21]$ and animal [21] musculature, whereas no significant differences were found for 4- versus 20-month-old rats in another study [30]. Increasing lipid content with age in our study is consistent with prior human $[12,22]$ and animal $[26,31]$ studies on the one hand and with increasing muscle T2 in the current study on the other hand.
Analysis of age- and diet-induced changes and focusing on detailed results of 16- and 21-month-old HFD and $\mathrm{CD}$ rats yields another interesting effect: nearly all measurement results are very similar for 16-month-old HFD and 21-month-old CD rats (see fig. 5). Thus, one might speculate that HFD induces 'accelerated muscle aging' in our animal model.

HFD does not inevitably lead to increased BW but should probably be regarded as a risk factor that merely increases the probability of abnormal body fat accumulation and obesity. On the other hand, CD does not automatically mean that all individual subjects remain lean with advancing age. This problem has not been sufficiently addressed in the literature yet in a rodent model. Therefore, we developed a stringent procedure for stratification of higher BW based on AUC analysis of the longitudinal weight gain. The results in the subgroups of heavier and normal-weight HFD and CD rats at the age of 16 months revealed that muscle quantity and quality are affected by both HFD and higher BW. Interestingly, higher BW alone did not cause a reduction of CSA in 16-month-old rats, but rather points to a BW-independent 'intrinsic' effect of the HFD.

As a very early work with an animal model for sarcopenia, our study has several limitations. First, using a clinical whole-body scanner resulted in some technical drawbacks for the current animal model when compared with dedicated animal MR systems - one limitation can be seen in separating IMCL and EMCL signal contributions in ${ }^{1} \mathrm{H}$-MRS. Assessment of T2 relaxation times in lean muscle and across the whole muscle was applied as a workaround in our study. Furthermore, the reason for prolonged muscle T2 under HFD and with advancing age needs additional investigation which might be based on imaging and/or histological examination. Second, the current study started with 16-month-old rats following a 10-month-long diet without taking into account earlier data of the animals. Assessment of baseline data would be necessary to exclude any bias. Third, some other, probably influencing, factors need to be considered in further studies. The physical activity of the animals has to be monitored and the influence of intensified or hindered activity might yield additional aspects. Gender is another important factor which has to be included in further studies because fat distribution is known to be significantly different in men and women [32]. It should be mentioned here that sarcopenia could be induced in a prior study in a transgenic mouse model in male, but not in female, mice [33]. 
In conclusion, this study strongly supports the hypothesis that HFD has a significant impact on quadriceps muscle quantity and quality in an animal model with male Sprague-Dawley rats. The main effects of HFD were a reduction of CSA and an increase in T2 relaxation time, which can be partially attributed to an increased fat content (predominantly IMCL) within the muscle. Both effects could be measured already at the age of 16 months and deteriorated with advancing age until the end point of the study (21 months) in HFD and
CD rats. This study might contribute to a better understanding of the pathogenesis of sarcopenia and might allow direct translation into the clinical geriatric setting.

\section{Acknowledgment}

This work was supported by the Robert Bosch foundation (L.C.B. \# 32.5.1141.0030.0) and the Bayerische Forschungsstiftung (project: FORMOsA).

\section{References}

1 Cooper C, Dere W, Evans W, Kanis JA, Rizzoli R, Sayer AA, Sieber CC, Kaufman JM, van Kan GA, Boonen S, Adachi J, Mitlak B, Tsouderos Y, Rolland Y, Reginster JY: Frailty and sarcopenia: definitions and outcome parameters. Osteoporos Int 2012;23:18391848.

2 Rolland Y, Lauwers-Cances V, Cristini C, van Kan GA, Janssen I, Morley JE, Vellas B: Difficulties with physical function associated with obesity, sarcopenia, and sarcopenic-obesity in community-dwelling elderly women: the EPIDOS (EPIDemiologie de l'OSteoporose) Study. Am J Clin Nutr 2009; 89:1895-1900.

-3 Zamboni M, Mazzali G, Fantin F, Rossi A, Di Francesco V: Sarcopenic obesity: a new category of obesity in the elderly. Nutr Metab Cardiovasc Dis 2008;18:388-395.

4 Li Z, Heber D: Sarcopenic obesity in the elderly and strategies for weight management. Nutr Rev 2012;70:57-64.

5 Cruz-Jentoft AJ, Baeyens JP, Bauer JM, Boirie Y, Cederholm T, Landi F, Martin FC, Michel JP, Rolland Y, Schneider SM, Topinková E, Vandewoude M, Zamboni M: Sarcopenia: European consensus on definition and diagnosis: report of the European working group on sarcopenia in older people. Age Ageing 2010;39:412-423.

6 Sakuma K, Yamaguchi A: Sarcopenic obesity and endocrinal adaptation with age. Int J Endocrinol 2013;2013:204164.

7 Turpin SM, Ryall JG, Southgate R, Darby I, Hevener AL, Febbraio MA, Kemp BE, Lynch GS, Watt MJ: Examination of 'lipotoxicity' in skeletal muscle of high-fat fed and ob/ob mice. J Physiol 2009;587:1593-1605.

8 Bollheimer LC, Buettner R, Pongratz G, Brunner-Ploss R, Hechtl C, Banas M, Singler K, Hamer OW, Stroszczynski C, Sieber CC, Fellner C: Sarcopenia in the aging high-fat fed rat: a pilot study for modeling sarcopenic obesity in rodents. Biogerontology 2012;13:609620.

-9 Visser M, Goodpaster BH, Kritchevsky SB, Newman AB, Nevitt M, Rubin SM, Simonsick EM, Harris TB: Muscle mass, muscle strength, and muscle fat infiltration as predictors of incident mobility limitations in well-functioning older persons. J Gerontol A Biol Sci Med Sci 2005;60:324-333.

10 Ratkevicius A, Joyson A, Selmer I, Dhanani T, Grierson C, Tommasi AM, De Vries A, Rauchhaus P, Crowther D, Alesci S, Yaworsky P, Gilbert F, Redpath TW, Brady J, Fearon KC, Reid DM, Greig CA, Wackerhage H: Serum concentrations of myostatin and myostatin-interacting proteins do not differ between young and sarcopenic elderly men. J Gerontol A Biol Sci Med Sci 2011;66:620626.

11 Delmonico MJ, Harris TB, Visser M, Park SW, Conroy MB, Velasquez-Mieyer P, Boudreau R, Manini TM, Nevitt M, Newman $\mathrm{AB}$, Goodpaster BH: Longitudinal study of muscle strength, quality, and adipose tissue infiltration. Am J Clin Nutr 2009;90:15791585.

12 Schwenzer N, Martirosian P, Machann J, Schraml C, Steidle G, Claussen CD, Schick F: Aging effects on human calf muscle properties assessed by MRI at $3 \mathrm{~T}$. J Magn Reson Imaging 2009;29:1346-1354.

13 Kuhlmann J, Neumann-Haefelin C, Belz U, Kramer W, Juretschke HP, Herling AW: Correlation between insulin resistance and intramyocellular lipid levels in rats. Magn Reson Med 2005;53:1275-1282.

14 Boettcher M, Machann J, Stefan N, Thamer C, Häring HU, Claussen CD, Fritsche A, Schick F: Intramuscular adipose tissue (IMAT): association with other adipose tissue compartments and insulin sensitivity. J Magn Reson Imaging 2009;29:1340-1345.

15 Schrauwen-Hinderling VB, Hesselink MKC, Schrauwen P, Kooi ME: Intramyocellular lipid content in human skeletal muscle. Obesity 2006;14:357-367.

16 Boesch C, Slotboom J, Hoppeler H, Kreis R: In vivo determination of intra-myocellular lipids in human muscle by means of localized ${ }^{1} \mathrm{H}-\mathrm{MR}$ spectroscopy. Magn Reson Med 1997; 37:484-493.

17 Boesch C, Machann J, Vermathen P, Schick F: Role of proton MR for the study of muscle lipid metabolism. NMR Biomed 2006; 19: 968-988.

18 Saukkonen T, Heikkinen S, Hakkarainen A, Häkkinen AM, van Leemput K, LipsanenNyman M, Lundbom N: Association of intramyocellular, intraperitoneal and liver fat with glucose tolerance in severely obese adolescents. Eur J Endocrinol 2010;163:413419.

19 Johannsen DL, Conley KE, Bajpeyi S, Punyanitya M, Gallagher D, Zhang Z, Covington J, Smith SR, Ravussin E: Ectopic lipid accumulation and reduced glucose tolerance in elderly adults are accompanied by altered skeletal muscle mitochondrial activity. J Clin Endocrinol Metab 2012;97:242-250.

20 Takahashi K, Takahashi HE, Nakadaira H, Yamamoto M: Different changes of quantity due to aging in the psoas major and quadriceps femoris muscles in women. J Musculoskelet Neuronal Interact 2006;6: 201-205.

21 Hatakenaka M, Ueda M, Ishigami K, Otsuka M, Masuda K: Effects of aging on muscle T2 relaxation time difference between fast- and slow-twitch muscles. Invest Radiol 2001;36: 692-698.

22 Nakagawa Y, Hattori M, Harada K, Shirase R, Bando M, Okano G: Age-related changes in intramyocellular lipid in humans by in vivo ${ }^{1} \mathrm{H}-\mathrm{MR}$ spectroscopy. Gerontology 2007;53: 218-223.

23 Bredella MA, Ghomi RH, Thomas BJ, Miller KK, Torriani M: Comparison of 3.0 T proton magnetic resonance spectroscopy short and long echo-time measures of intramyocellular lipids in obese and normal-weight women. J Magn Reson Imaging 2010;32: 388-393.

24 Lexell J: Human aging, muscle mass, and fiber type composition. J Gerontol A Biol Sci Med Sci 1995;50:11-16.

25 Gloor M, Fasler S, Fischmann A, Haas T, Bieri O, Heinimann K, Wetzel SG, Scheffler K, Fischer D: Quantification of fat infiltration in oculopharyngeal muscular dystrophy: comparison of three MR imaging methods. J Magn Reson Imaging 2011;33:203-210. 
26 De Feyter HM, Lenaers E, Houten SM, Schrauwen P, Hesselink MK, Wanders RJ, Nicolay K, Prompers JJ: Increased intramyocellular lipid content but normal skeletal muscle mitochondrial oxidative capacity throughout the pathogenesis of type 2 diabetes. FASEB J 2008;22:3947-3955.

27 van den Broek NM, Ciapaite J, De Feyter HM Houten SM, Wanders RJ, Jeneson JA, Nicolay $\mathrm{K}$, Prompers JJ: Increased mitochondrial content rescues in vivo muscle oxidative capacity in long-term high-fat-diet-fed rats. FASEB J 2010;24:1354-1364.
8 Beha A, Juretschke HP, Kuhlmann J, Neumann-Haefelin C, Belz U, Gerl M, Kramer W, Roden M, Herling AW: Muscle type-specific fatty acid metabolism in insulin resistance: an integrated in vivo study in Zucker diabetic fatty rats. Am J Physiol Endocrinol Metab 2006;290:E989-E997.

29 Conley KE, Cress ME, Jubrias SA, Esselman PC, Odderson IR: From muscle properties to human performance, using magnetic resonance. J Gerontol A Biol Sci Med Sci 1995;50: $35-40$.

30 Nicolato E, Farace P, Asperio RM, Marzola P, Lunati E, Sbarbati A, Osculati F: Dynamic contrast-enhanced magnetic resonance imaging of the sarcopenic muscle. BMC Med Imaging $2002 ; 2: 2$.

- 31 Neumann-Haefelin C, Kuhlmann J, Belz U, Kalisch J, Quint M, Gerl M, Juretschke HP, Herling AW: Determinants of intramyocellular lipid concentrations in rat hindleg muscle. Magn Reson Med 2003;50:242-248.
32 Machann J, Thamer C, Stefan N, Schwenzer NF, Kantartzis K, Häring HU, Claussen CD, Fritsche A, Schick F: Follow-up whole-body assessment of adipose tissue compartments during a lifestyle intervention in a large cohort at increased risk for type 2 diabetes. Radiology 2010;257:353-363.

33 Reisz-Porszasz S, Bhasin S, Artaza JN, Shen R, Sinha-Hikim I, Hogue A, Fielder TJ, Gonzalez-Cadavid NF: Lower skeletal muscle mass in male transgenic mice with muscle-specific overexpression of myostatin. Am J Physiol Endocrinol Metab 2003;285:E876-E888. 